\title{
Acoustic Mapping Velocimetry (AMV) for in-situ bedload transport estimation
}

\author{
S. Baranya \\ Budapest University of Technology and Economics, Budapest, Hungary \\ M. Muste \\ IIHR-Hydroscience and Engineering, The University of Iowa, Iowa City, Iowa, United States of America
}

D. Abraham

USACE-ERDC-CHL, Vicksburg, Mississippi, United States of America

T. C. Pratt

USACE-ERDC-CHL, Vicksburg, Mississippi, United States of America

ABSTRACT: Despite the importance of sediment transport processes in large rivers, the measurement of sediment transport rate in the in-situ, especially bedload, is difficult, costly and time consuming using conventional methods. In this paper, a novel indirect bedload estimation methodology is presented that is based on the Acoustic Mapping Velocimetry (AMV). AMV is a combination of acoustic and imaging techniques that provides 2D bedform velocity maps. As such, it can only be used if bedload is represented by bedform migration. This paper illustrates the applicability of the bedload estimation method using as test case a section of the Ohio River in the United States. Repeated measurements of the bathymetry provided by multi-beam echo sounder serve as input data for AMV. Cross-sectional distributions of bedload transport rates obtained with AMV are compared with the estimates provided by another non-intrusive technique, ISSDOTv2, developed by the US Army Corps of Engineers. The good agreement between the results with the two different methods is encouraging and suggests further field tests covering a wider range of hydro-morphological situations.

\section{INTRODUCTION}

Knowledge of sediment transport processes in rivers is of great importance due to the wide range of fields influenced by sediment motion. The transport, deposition and resuspension of sediment grains affect the quality of aquatic ecosystems, fluvial navigation, drinking water supply, hydropower, flood risk as well as the natural formation of coastal areas. It is, therefore, crucial to gain knowledge on the quantity and also the quality of the sediments moving downstream in the river. As to the bedload transport in rivers, it is very problematic to use conventional, i.e. direct, samplers, such as pressure-difference samplers (e.g., Helley and Smith; 1971), to estimate the transport rate, especially in large rivers. The movement of sediments at the riverbed shows complex spatio-temporal behavior and therefore the samples collected at a point during short sampling times are often not representative. Moreover, in some situations direct sampling is simply not feasible due to the measurement environment. As a result, indirect bedload estimation methods using acoustic techniques have increasingly been tested and many of them show promising results. When bedload transport consists of migrating bedforms, tracking their movement and applying appropriate algorithms can directly yield the bedload transport rates. Several studies have been performed processing bed profiles from repeated longitudinal bathymetric surveys and estimating the bedform velocity (e.g. Simons et al., 1965: Engel and Lau, 1980; Kostaschuk et al., 1989; Dinehart, 2002; Gray et al., 2010). These applications, however, consider the bedload transport as a 1D process and do not take into account the lateral sediment movement. An improved methodology using data from repeated channel bed geometry surveys was reported by Abraham et al. (2011) using acoustic-based surveys, called ISSDOTv2. Their method consists of measuring rates of bed transport using time-sequenced bathymetric data. The method was validated in a laboratory comparing measured rates of bed scour with direct measurements of bedload transport. The method has also been used at numerous field measurement sites (see for example Abraham et al 2015a, Abraham et al 2015b, Heath et al 2015).

In this paper we use the so-called Acoustic Mapping Velocimetry (AMV) technique to gain information on bedform migration (Muste et al, 2015). The method combines components and processing protocols from two contemporary non-intrusive approaches: acoustic and image-based. The bedform mapping is conducted with acoustic surveys while the estimation of the velocity of the bedforms is obtained with processing techniques pertaining to image-based velocimetry. The implementation of this technique produces a whole-field velocity map associated with the multi-directional bedform movement.

In this study the field testing of the method is presented. We use bathymetric data, collected in the 
Ohio River, where repeated multibeam echo sounder (MBES) surveys were carried out. In the following discussion we introduce the AMV method, the field measurements, the flow conditions as well as the AMV based bedload transport estimation. The latter will be compared with the estimates provided by another non-intrusive technique, ISSDOTv2.

\section{AMV METHODOLOGY}

The implementation of the AMV entails two phases. First, acoustic maps are created as a continuous depth-data layer covering the target area of the river bottom. Second, the acoustic maps are converted to an "image-equivalent" through resampling of the raw information. The obtained images are subsequently processed with image velocimetry techniques. Bedload rates are obtained by combining the outcomes from both acoustic mapping acquired in step 1 and the velocity fields obtained in step 2 with analytical relationships.

The acoustic maps are 3D representations of the bed geometry. In this study the maps were created using repeated MBES surveys as will be introduced later on. There are important considerations that need attention prior to mapping with acoustic survey instruments. Similar to the recording of a photographic snapshot (whereby all the image pixels are captured at the same instant), the acoustic map should ideally contain depth measurements acquired simultaneously over the whole mapped area. This is, however, impossible in wide rivers where the river bed is usually scanned along parallel swaths, where the swath width is limited according to the water depth and the device used. Given that the depth measurements are not acquired simultaneously, the obtained map will be unavoidably affected by the movement of the bedform during the acquisition of the depth measurements. In this study, for instance, the survey of a $1 \mathrm{~km}$ long swath took 4-6 minutes and the mapping of the whole cross-section took approximately 90 minutes. Considering a bedform movement speed in the order of $0.001 \mathrm{~m} / \mathrm{s}$, the scanning of one longitudinal line can be assumed as an instantaneous image of the river bed. The bedload estimation in this study is based on individual swaths and therefore the time lag between the successive swaths will not affect the calculations. It has to be noted, however, that the accurate construction of the maps require development of a measurement protocol that gives full consideration to the characteristics of the instruments, the size of the target map, and the bedform geometry to be mapped and their dynamics. As intriguing as it sounds, the last consideration needs to be known even before conducting the mapping. From this perspective, before acquiring acoustic maps in a new situation, preliminary measurements should be acquired to roughly assess characteristics of the bedforms (e.g., dune wavelength, other geometrical characteristics) and their dynamics. Based on these preliminary measurements, the spatial coverage of the measurement and the associated protocols can be accordingly adjusted. Once acquired, the acoustic maps are typically presented as color-coded or iso-contour maps. The color maps are 2.5-dimensional representations of the domain, whereby the range of colors represent the bed elevation.

The estimation of the bedform velocity maps is in fact based on the particle image velocimetry concepts (Adrian, 1991). Essentially, image velocimetry estimates probable displacements of recognizable patterns embedded in a sequence of images, bedforms in this case. The displacements are determined using a statistical approach whereby a pattern matching technique is applied to image intensity distribution in a series of images (Adrian, 1991; Fujita et al., 1998). The analysis is made successively over the entire imaged area using small interrogation areas (IA) covering the area subjected to measurement, as illustrated in Figure 2a. The similarity index for patterns enclosed in a small IA in an image is calculated for the same-size window within a larger Search Area (SA) selected in the subsequent image. The selection of IA and SA is guided by heuristic rules of thumb (e.g., Adrian, 1991; Raffel et al., 1998) but typically they are optimized by trial and error for a given application.

The similarity index used for image processing in this paper is estimated using the spatial crosscorrelation (Fujita et al., 1998) applied to a pair of acoustic maps (shown in figure 2.b). Fujita's algorithm is based on an image velocimetry approach developed by Fincham and Spedding (1997) that is favorable for this application as the cross-correlation is applied to grey-level patterns in the image rather than point clusters (Muste et al., 2008). The Fincham and Spedding algorithm is similar to the crosscorrelation algorithm applied to linear seafloor features embedded in the acoustic maps developed by Duffy (2006). The result of image velocimetry processing is a distributed velocity field uniformly covering the acoustically mapped area (see Figure 2.c).

Prior to applying image velocimetry to acoustic maps, the conversion of the acoustic maps to graylevel maps is needed. For this purpose, the depth measurements obtained as described above and color-mapped using red, green and blue are converted into gray-colored pixels using a scale from 0 to 255 . The interpolated color maps are converted in pixel coordinates by successively sampling the entire image. The resolution of the pixel image is decided by the user, but it should be commensurate with the spatial resolution of the mapping and the scale of the bedform spatial features.

The whole-field velocity distribution associated with acoustic maps can be used in conjunction with 
analytical methods for evaluating bedload rate estimates. The method selected for this study is based on the continuity equation applied to bedform movement (Exner, (1925):

$(1-p) \frac{\partial y}{\partial t}+\frac{\partial q_{b}}{\partial x}=0$

where $p=$ porosity of the river bed, $y=$ bed elevation, $t=$ time, $q_{\mathrm{b}}=$ volumetric bedload transport rate per unit width and $\mathrm{x}=$ longitudinal distance. Assuming a steady uniform flow and that the dunes are in equilibrium, Simons et al. (1965) introduced the following formula for the volumetric bedload per unit width:

$q_{b}=(1-p) V_{D} \frac{\Lambda}{2}$

where $V_{\mathrm{D}}$ is the bedform velocity and $\Lambda$ is the bedform height. The bedload transport by weight can then be estimated by multiplying $q_{\mathrm{b}}$ by the density of the sediment and integrating it along the given crosssection of the river.

The estimation of bedload rates using Equation

(2) entails the following steps:

1. acquisition creation of a sequence of acoustic maps (preferably at equal time steps).

2. quantification of bedform dynamics (i.e. calculation of $2 \mathrm{D}$ bedload velocity fields within the mapped area)

3. Determination of characteristic bedform height within the mapped area

4. estimation of bedload transport using Equation (2) in conjunction with information obtained in steps 2 and 3.

\section{FIELD MEASUREMENTS}

The study site chosen for this research was a one $\mathrm{km}$ long reach of the Ohio River in the US, at River mile 971, close to the confluence of the Mississippi river at Cairo, IL. The river can be characterized with an average width of $1000 \mathrm{~m}$, an average depth of $10 \mathrm{~m}$ and a mean annual discharge of $8000 \mathrm{~m}^{3} / \mathrm{s}$. The bed material is medium sand with a typical $d_{50}$ of 0.3 $\mathrm{mm}$. Large bedforms are present at this section of the Ohio River with a characteristic wave height of $1.5 \mathrm{~m}$ and wave length of $150 \mathrm{~m}$. The field surveys were performed by the US Army Corps of Engineers on March 27, 2013 in high water regime at a flow discharge of $14000 \mathrm{~m}^{3} / \mathrm{s}$.

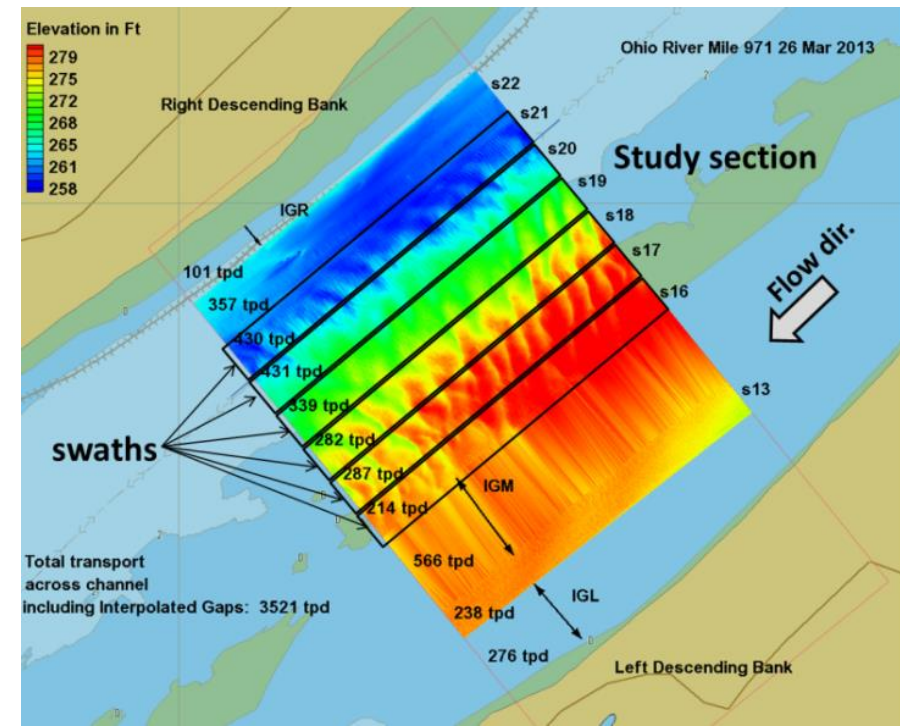

Figure 1. Bed geometry map or Ohio River at rm 971. Color palette indicates bed elevation levels.

The comprehensive field expedition consisted of repeated MBES scanning, ADCP measurements, bed material and suspended sediment samplings. The bed geometry mapping was carried out along eight longitudinal swaths as shown in Figure 1 through the use of a $250 \mathrm{KHz}$ Geoswath echo sounder. The survey boat was RTK GPS positioned and compensated for pitch, heave and roll. Horizontal accuracy was stated as $+/-2 \mathrm{~cm}$ and vertical resolution of bathymetric elevations was approximately $3 \mathrm{~cm}$ in 50 meter of water. This allowed vertical bathymetric changes of more than $5 \mathrm{~cm}$ to be recorded and used in determining the elevation change between two profiles taken at two different times over the same spatial location. The mapping was repeated four times. Out of the eight transects six of them (s16s21) were analyzed with the AMV method, where bedforms were observed. Table 1 summarizes the starting times of the measurement for each swath. This information was used to define the delta time between swath pairs for the bedform velocity calculations. The time stamps show a quite even measurement time of 4-6 minutes per swath and a total time of 80-140 minutes for the entire cross section. Four ADCP transect measurements were acquired at $\mathrm{Rm} 971$ using a bin size for the velocity measurements of $0.25 \mathrm{~m}$ and a sampling frequency of 2.5 $\mathrm{Hz}$.

Table 1. Starting times of MBES surveys for individual swaths.

\begin{tabular}{l|l|l|l|l} 
& Round 1 & Round 2 & Round 3 & Round 4 \\
\hline s16 & $15: 40$ & $17: 06$ & $18: 46$ & $20: 58$ \\
\hline s17 & $15: 36$ & $17: 01$ & $18: 41$ & $20: 53$ \\
\hline s18 & $15: 29$ & $16: 54$ & $18: 35$ & $20: 47$ \\
\hline s19 & $15: 25$ & $16: 50$ & $18: 31$ & $20: 43$ \\
\hline s20 & $15: 18$ & $16: 44$ & $18: 24$ & $20: 37$ \\
\hline s21 & $15: 11$ & $16: 39$ & $18: 19$ & $20: 32$ \\
\hline
\end{tabular}

As discussed above, the AMV method to estimate bedload transport is based on the bedform migration 
and excludes the effect of sediment resuspension close to the river bed. It was, therefore, important to reveal if significant suspended sediment transport occurred during the field tests or not. The Rousenumber is a suitable indicator to separate suspended and bed material transport, therefore the estimation of this parameter was carried out based on measured flow velocity data. First, the cross-sectional distribution of the bed shear velocity was calculated. The estimation was done assuming a logarithmic velocity profile close to the river bed. Using the least-squares method the analytical wall function was fitted on the ten lowermost points of the spatially averaged velocity distribution (see e.g. Baranya, 2010):

$$
\frac{U}{u_{*}}=\frac{1}{\kappa} \ln \left(\frac{30 y}{k_{s}}\right)
$$

where $U=$ flow velocity, $u_{*}=$ bed shear velocity, $\kappa=$ von Kármán constant $(0.41), y=$ distance from river bed, $k_{\mathrm{s}}=$ roughness height. The Rouse number, $Z_{\mathrm{R}}$ was calculated based on the bed shear velocity and the settling velocity $\left(w_{\mathrm{s}}\right)$ of the mean grain size of the bed material:

$$
Z_{R}=\frac{w_{s}}{\kappa u_{*}}
$$

Figure 2 shows the cross-sectional distribution of the estimated Rouse-number from the four ADCP crossings as well as the average of the four. The measured depth values are also plotted. It can be clearly seen that the Rouse-number values fall in the range of 4-10, indicating no suspension (suspension occurs at $Z_{\mathrm{R}}<2.5$; Rouse, 1939). Lowest $Z_{\mathrm{R}}$ values appear in the deepest zones, where the highest velocities and highest bed shear stress values characterize the flow. In overall, the distribution is reasonably homogeneous with an average value around 7, showing slight increasing towards the left bank. Based on this assessment it can be stated that at the actual flow conditions no significant suspension takes place and so the herein applied bedload estimation methodology can well represent the total bedload.

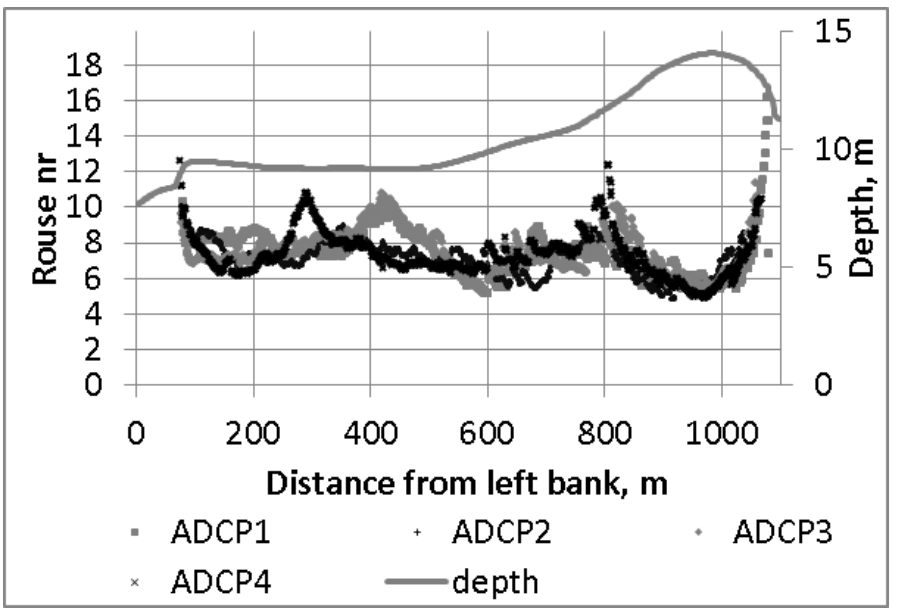

Figure 2. Cross-sectional distribution of Rouse-number estimated from ADCP velocities and flow depth (see text for more details).

\section{RESULTS}

\subsection{Estimation of the bedform velocity maps}

Using the above described image processing procedure, 2D bedform velocity maps were created for each swath separately and for each measurement pair, i.e. between the first and second, second and third, third and fourth MBES survey, respectively. Also, a comprehensive sensitivity analysis was performed to find the optimal parameters of the AMV procedure. The following settings were studied: image resolution, size of interrogation area and crosscorrelation threshold. Considering the accuracy, the spatial resolution and extension of the velocity maps we used an image resolution of $1 \mathrm{~m}$, an IA size of 32 pixels, and a correlation coefficient limit of 0.8 .

The bedform migration velocity maps, plotted for each swath in Figure 3, are based on the first two MBES surveys. Two similar maps were obtained using the second and third, and the third and fourth scannings, respectively. The bedform migration velocities range between $0.0002-0.0004 \mathrm{~m} / \mathrm{s}$, which is $0.72-1.44 \mathrm{~m} / \mathrm{h}$. This migration speed allows us to consider a 4-5 minute long swath measurement as an instantaneous map of the river bed. By considering a 80-140 minutes interval between two successive measurement rounds, the bedform displacement is of the order of meters, which is suitable for the image processing method. The migration velocities display larger velocities towards the right bank which is expected as the more intense bedform movement is typically associated with deeper areas of the river.

Besides the cross-sectional variation of the bedform velocity, there are other significant changes that can be observed. A periodic variation of low and high velocity patches can be observed for each swath which is the direct result of the location of the crests and troughs on the bedforms. As expected, higher bedload transport rates appear on the dune crests and lower transport rates on the trough. The unique ad- 
vantage of the AMV method is that it reveals both the bedform geometry as well as the 2D velocity vector field simultaneously using the one set of raw measurements (see Fig. 3, bottom). This plot substantiates the 2D nature of the maps with strong nonhomogeneities in the direction of bedform migration. The bedload estimation procedure used in the present analysis considers only the streamwise movement of the bedform. A more refined version of the analysis can take into considerations the lateral movement displayed by the bedform as the data needed for evaluation is readily available.

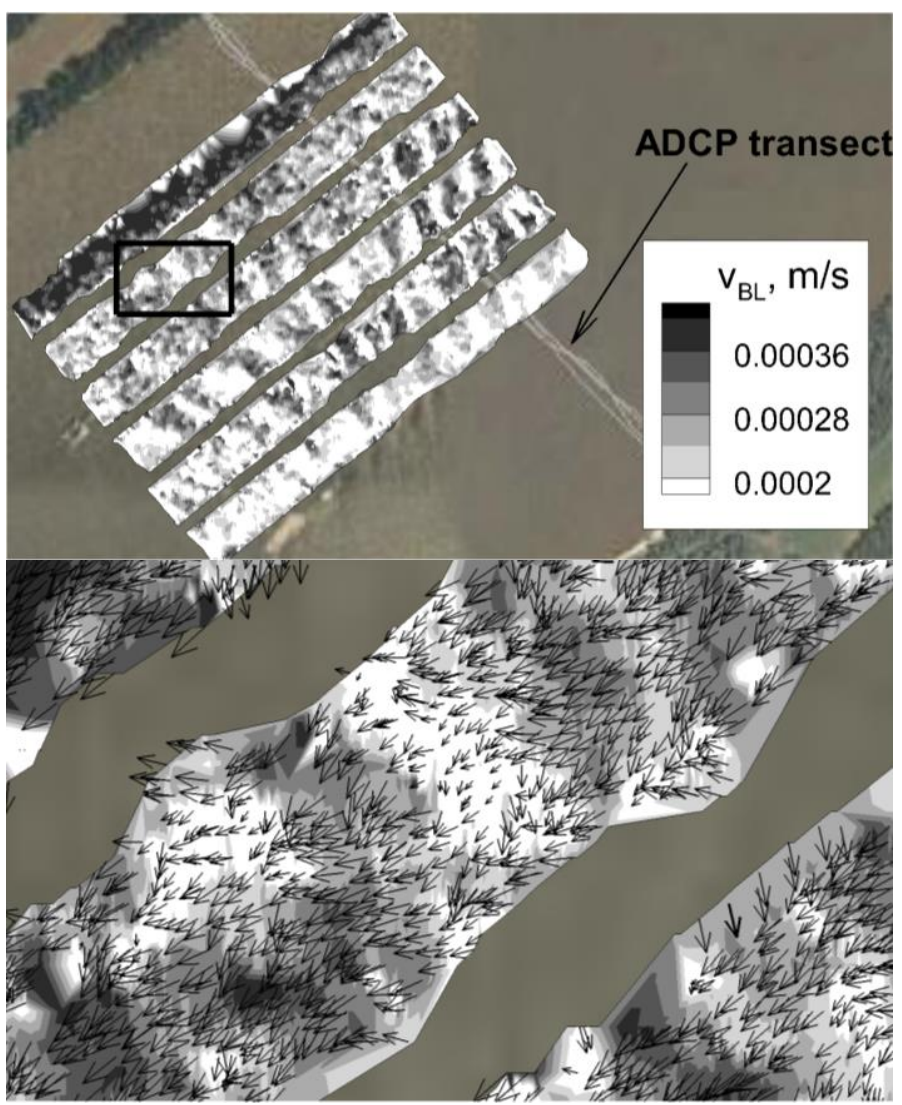

Figure 3. Top: calculated bedform migration velocity maps for all the swaths (based on first and second MBES surveys); Bottom: bedform velocity vectors for the area indicated above.

\subsection{Estimation of bedform geometry}

Quantification of the bedload transport requires the determination of the bedload layer thickness. Typically, in case of bedform migration the layer thickness is assumed to be the half of the average bedform height (see Eq. 2). The estimation of this parameter in complex bed geometry situations is not straightforward. In this study a bedform analysis was performed along the centerline of each individual swath to provide input data for the Exner-equation. For this purpose, the so called Bedform Tracking Tool software (van der Mark et al., 2008) was used. This tool performs a spectral analysis on longitudinal profiles to calculate typical bedform geometry parameters. A sample estimation obtained with the tool for a longitudinal profile over swath s18is shown in Figure 4.
The complex geometrical structure of the bed geometry is well illustrated in this figure by the large dunes covered by ripples. The spectral analysis indicates that the average height of the large bedforms is $1.5 \mathrm{~m}$, whereas the heights of the ripples are around $0.2 \mathrm{~m}$. Taking into account the spatial resolution of the AMV method, the bedform velocity determined with image velocimetry corresponds to the movement of the ripples, hence the bedload transport estimated is associated with these type of bedforms. The contribution of the large dune movements to the total bedload transport, however, remains to be clarified through future studies. The relationship between the movement of the large and small scale bedform and the actual rates of sediment transport defined through analytical means or alternative measurement are issues that are beyond the scope of the present study.

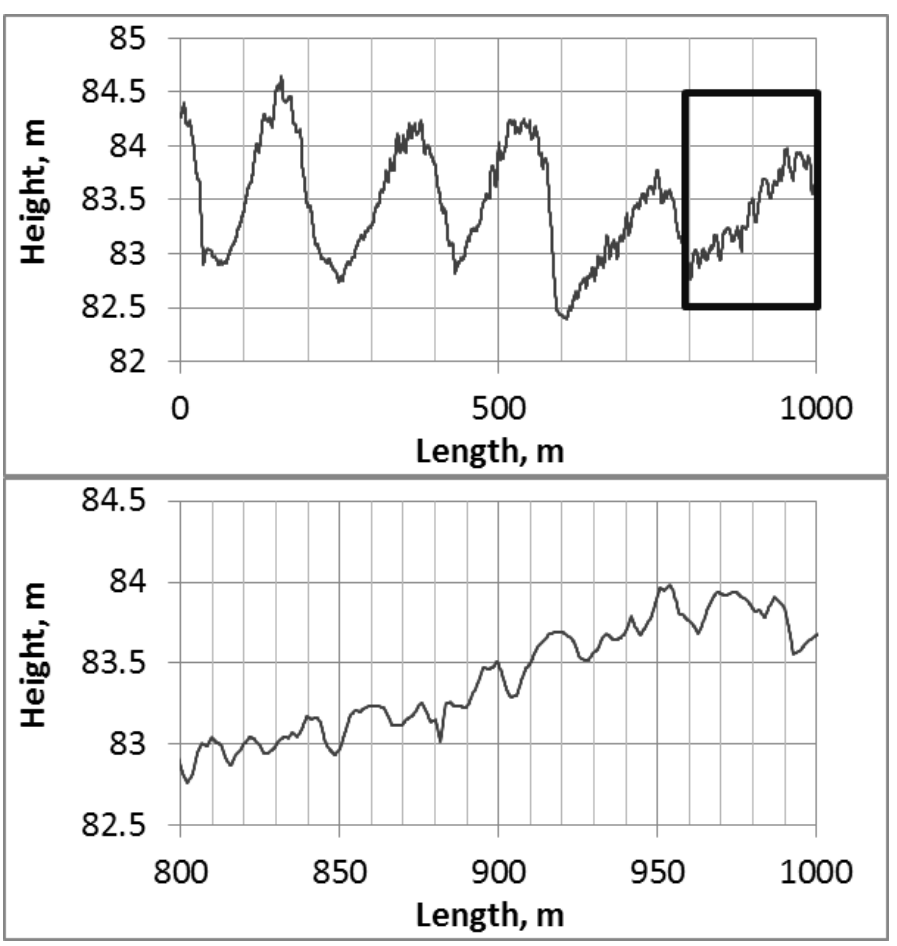

Figure 4. Top: Longitudinal profile of centerline at S18. Bottom: subsection of the longitudinal profile for the area indicated above.

Using the conceptual approach described above, the characteristic height of the ripple migration was estimated for each swath. The range for the ripples heights varies between 0.15 and $0.25 \mathrm{~m}$ for the study area. No strong correlation was found between bedform height and bedform migration velocity as they appear in different parts of the cross section. For example, observations on swath s21 indicate that the most intensive ripple migration takes place close to the right bank (in the deepest cross section area), while the tallest ripples occur towards the centerline. 


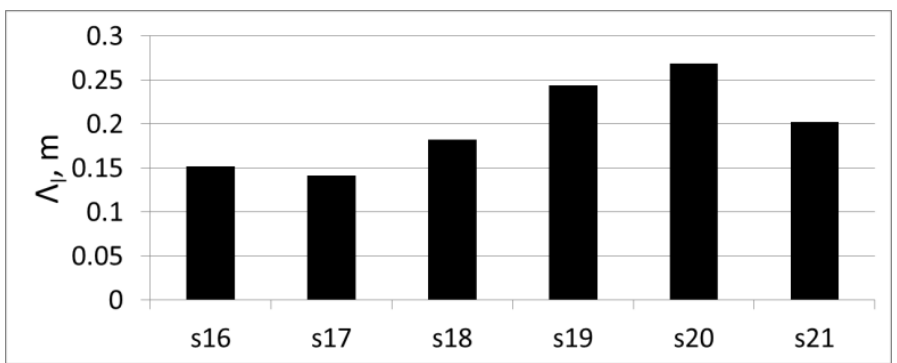

Figure 5. Characteristic bedform height for individual swaths.

\subsection{Estimation of bedload transport}

Spatially-averaged bedload transport rates for each individual swath were estimated using the spatiallyaveraged bedform migration velocities and the associated characteristic bedform height. Only the streamwise component of the migration velocities was used, as this is the one contributing to the bedload transport. Using the four successive acoustic maps the temporal variation of the bedload transport could also be obtained. The calculated bedload rates for each swath and for each acoustic map pair are plotted in Figure 6 (top). These estimates are compared with those provided by Abraham et al. (2010) method in Figure 6 (bottom).

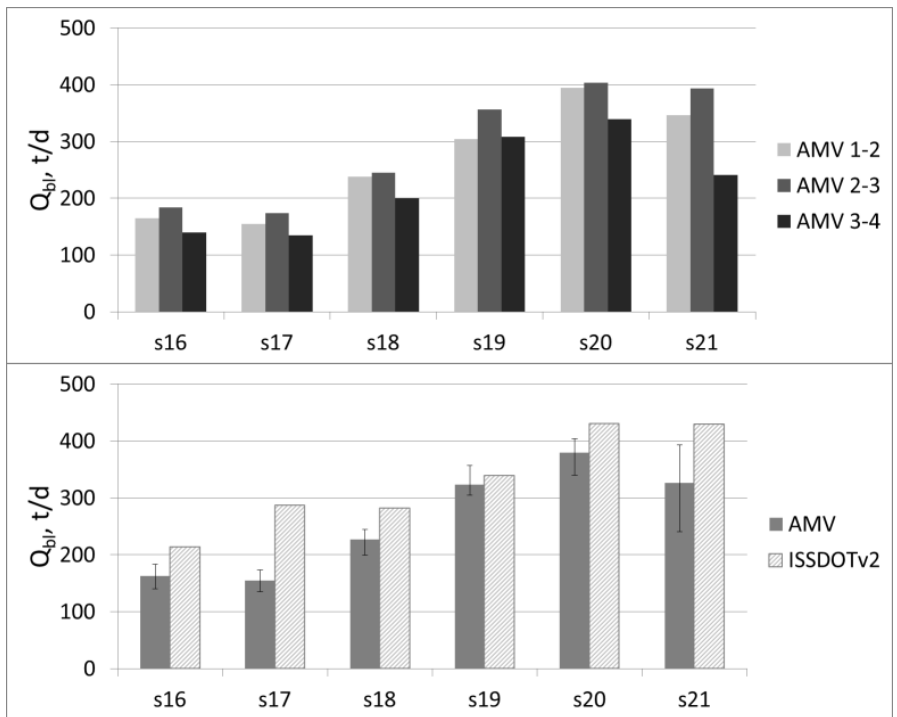

Figure 6. Top: Cross-sectional distribution of the bedload transport rates using the AMV method for each acoustic map pair; Bottom: Time-averaged bedload transport rates from AMV method (error bars indicate temporal variation) and from the ISSDOTv2 (Abraham et al., 2010) method.

The bedload transport rates were estimated for each 100-meter wide swath (in tons/day). The lowest transport rates occur on the left side of the maps with values around 160 tons/day. Increasing rates can be observed towards the deeper cross-section areas with bedload transport rates up to $\sim 350$ tons/day. The significant cross-sectional variation of the sediment transport substantiates the need to estimate rates over the whole cross-section. This is in contrast with the sampling methodology of the conventional methods for estimating bedload characteristics whereby samples are collected over limited areas or at a point in the cross section. The variation of the rates in time is not significantly different between the pair of images used for estimating the bedload rates (differences of up to $20 \%$ in the most active transport area on the right banks, but with most for all the other swaths with that does not exceed $10 \%$ ). The practical implications of this finding is that acceptable accuracy can be expected using the AMV method applied to just one pair of acoustic maps.

The comparison of the bedload transport rates calculated with the AMV and ISSDOTv2 tool reveals good agreement overall and consistent trends over the cross section. The differences between the two methods are up to $20 \%$, with bedload transport estimates provided by ISSDOTv2 consistently higher as indicated in Fig. 7. The most probably explanation for the difference is the fact that AMV estimates rates strictly along the streamwise direction, while ISSDOTv2 include changes of volume over in both streamwise and spanwise directions. Nevertheless, the agreement between the two indirect estimation procedures is relevant indicating that acoustic mapping promises major improvements in the efficiency of the bedload transport estimation in large sand bed rivers where any of the intrusive methods would require considerably larger sampling efforts.

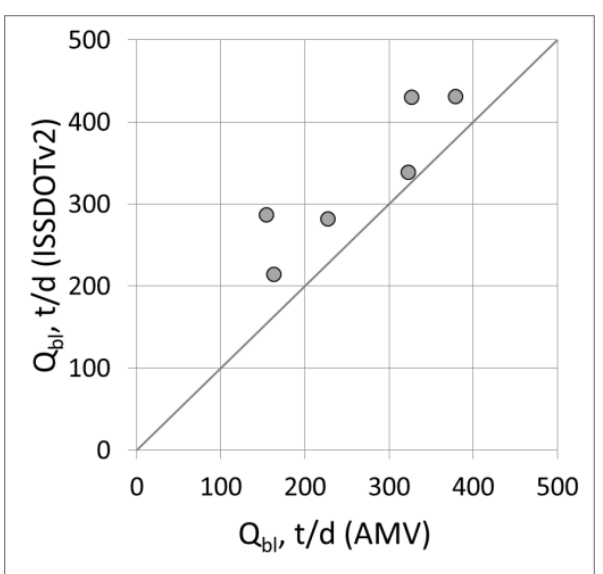

Figure 7. Comparison of calculated bedload transport rates from AMV and ISSDOTv2 methods.

\section{CONCLUSIONS AND FUTURE WORK}

The paper presents the first field implementation of the AMV for estimation of the bedload rates. Multibeam echo sounder (MBES) survey data acquired in the Ohio River (in the US) was used for this purpose. Validation of the AMV results was done using the Abraham et al. (2010) method as reference. The agreement between the results obtained with the two methods is notable for this type of complex measurements, confirming that the proposed AMV method is a good candidate for non-intrusively estimating bedload transport measurements in large rivers with bedload dominated by bedform migration. 
The MBES was proven to be an excellent source of raw data for the AMV method despite that the two methods were developed independently. The synergy between the two techniques makes them well fit for in-situ implementation. First, not only that MBES has a native spatio-temporal resolution suitable for the AMV, but its sampling efficiency over large scales is unparalleled by the capabilities of any other available method or instrument. Given that the current field measurement practice bundles together MBES and ADCP acquired simultaneously, allows connecting the bedload movement with the water dynamics in the water column. Consequently, new areas of explorations can be approached such as the characterization of sheet flows (e.g. Rennie et al., 2002; Rennie et al., 2007; Jamieson et al., 2011) and the measurement of the suspended sediment in the water column simultaneously with the acquisition of the 3D velocity field (e.g. Guerrero et al., 2011; Guerrero et al., 2012). This pairing of instruments, currently used for much less ambitious tasks, has the potential to document flow and sediment transport as previously was not conceivable outside of laboratory conditions.

\section{ACKNOWLEDGEMENTS}

We acknowledge the funding of Sándor Baranya from the Dr. Imre Korányi Scholarship and the János Bolyai fellowship of the Hungarian Academy of Sciences. The second author was supported by grant 11-TI-C06 of the Ministry of Land, Infrastructure and Transport (Korea).

\section{REFERENCES}

Abraham, D., Pratt, T., and Sharp, J. (2010). "Measuring bedload transport on the Mis souri River using time sequenced bathymetric data." $2^{\text {nd }}$ Joint Federal Interagency Conf., Las Vegas, NV.

Abraham, D., Kuhnle, R., and Odgaard, A. (2011). "Validation of Bed-Load Transport Measurements with TimeSequenced Bathymetric Data." J. Hydraul. Eng., 137(7), 723-728.

Abraham, D., McAlpin, T., May, D., Pratt, T., Shelley, J. 2015a. "Update on ISSDOTv2 Method for Measuring Bed-load Transport with Time Sequenced Bathymetric Data." Proceedings of the $10^{\text {th }}$ Federal Interagency Sedimentation Conference, April 2015, Reno, Nevada, USA.

Abraham, D. D., Clifton, N. D. and T. C. Pratt. 2015b. "Numerical Sedimentation Study of Shoaling on the Ohio River near Mound City, Illinois. USACE ERDC/CHL Technical Report No. TR-15-12

Adrian, R.J. (1991), Particle-Imaging Techniques for Experimental Fluid Mechanics. Ann Rev Fluid Mech, (23), pp. 261-304.

Dinehart, R.L. (2002), Bedform movement recorded by sequential single-beam surveys in tidal rivers. J. Hydrol. 258, 1-4, 25-39, DOI: /10.1016/S0022- 1694(01)00558-3.

Duffy, G. P. (2006), Bedform Migration and Associated Sand Transport on a Banner Bank: Application of Repetitive
Multibeam Surveying and Tidal Current Measurement to the Estimation of Sediment Transport. PhD Thesis. The University of New Brunswick.

Engel, P., and Y.L. Lau (1981), Bed load discharge coefficient. J. Hydraul. Div. 107, 11, 1445-1454.

Exner, F. M. (1925), U* ber die Wechselwirkung zwischen Wasser und Geschiebe in Flüssen (in German), Sitz. Acad. Wiss. Wien Math. Naturwiss. Abt. 2a, 134, 165 - 203.

Fincham, A. M. and G.R. Spedding (1997), Low-cost, High Resolution DPIV for Measurement in Turbulent Fluid Flows. Exp. in Fluids, 23, pp. 449-462

Fujita, I., Muste, M., and Kruger, A. (1998), Large-Scale Particle Image Velocimetry for Flow Analysis in Hydraulic Applications. J. Hydr. Res., 36(3), pp. 397-414.

Gray, J.R., Laronne, J.D., and Marr, J.D.G. (2010), Bedloadsurrogate monitoring technologies. U.S. Geol.Surv. Scientific Investigations Report 2010-5091, 37p.

Guerrero, M., Szupiany, R.N., and Amsler, M. (2011), Comparis on of acoustic backscattering techniques for suspended sediments investigation. Flow Measurement and Instrumentation, 22, 5, 392-401.

Guerrero, M., Rüther, N., and Szupiany, R.N. (2012), Laboratory validation of acoustic Doppler current profiler (ADCP) techniques for suspended sediment investigations. Flow Measurement and Instrumentation, 23, 1, 40-48.

Heath, R. E., Brown, G. L., Little, C. D., et al. 2015. "Old River Control Complex Sedimentation Investigation." USACE ERDC/CHL Technical Report No. TR-15-8.

Helley, E.J., and Smith, W. (1971), Development and Calibration of a Pressure-difference Bedload Sampler. U.S. Geological Survery Open-file Report, 1-18.

Jamieson, E.C., Rennie, C.D., Jacobson, R.B., and Townsend, R.D. (2011), Evaluation of ADCP bed velocity in a large sand bed river: Moving versus stationary boat conditions. Journal of Hydraulic Engineering (ASCE), 137:1064-1071.

Kostaschuk, R. A., M. A. Church, and J. L. Luternauer (1989), Bedforms, bed material, and bedload transport in a saltwedge estuary: Fraser River, British Columbia. Can. J. Earth Sci., 26, 1440- 1452.

Muste, M., Fujita, I., and Hauet, A. (2008), Large-Scale Particle Image Velocimetry for Measurements in Riverine Environments. Special Is sue on Hydrologic Measurements, Water Resources Research, 44, W00D19, doi: 10.1029/2008WR006950

Muste, M., Baranya, S., Kim, D, Ho, H-C., Tsubaki, R., Tsai, D., and Law, D. (2015). Acoustic Mapping Velocimetry Proof-of-Concept Experiment, Proceedings 36th IAHR World Congress, June 28-July 3, 2015, Delft- The Hague, The Netherlands

Raffel, M., Willert, C.E., and Kompenhans, J. (1998), Particle Image Velocimetry: A Practical Guide. Springer, New York, NY. USA.

Rennie, C. D., Millar, R. G., and Church, M. A. (2002), Measurement of bed load velocity using an acoustic Doppler current profiler. J. Hydraulic Eng., 128(5), 473-483.

Rennie, C. D., Rainville, F., and Kashyap, S. (2007), Improved estimation of ADCP bedload velocity using a real time Kalman filter. Journal of Hydraulic Engineering, 133(12),1337-1344. Special Issue on Acoustic Measurements in Rivers.

Simons, D.B., Richardson, E.V. and Nordin, C.F. (1965). "Bedload Equation for Ripples and Dunes," U.S. Geological Survey Profes sional Paper 463-H, pp. 1-9.

Van der Mark C.F., Blom, A. and Hulscher, S.J.M.. (2008). "Quantification of Variability in Bedform Geometry," $J$. Geophys. Res, 113, F03020; doi:10.1029/2007JF000940. 